Boguslaw Kaczmarski, Pedagogical University of Kielce

Piotrkow Trybunalski Branch, Poland

\title{
ON THE SETS WHERE A CONTINUOUS FUNCTION HAS INFINITE ONE-SIDED DERIVATIVES
}

\begin{abstract}
In the present paper I give a characterization by help of measure and Borel classes of the set of points at which the continuous function possesses an infinite one-sided derivative. The main theorem is as follows. Let $E_{1}$ and $E_{2}$ be disjoint subsets of $\mathbb{R}$. There exists a continuous function $f: \mathbb{R} \rightarrow \mathbb{R}$ such that $E_{1}=\left\{x: f_{+}^{\prime}(x)=+\infty\right\}$ and $E_{2}=\left\{x: f_{+}^{\prime}(x)=-\infty\right\}$ if and only if (i) $E_{1}$ and $E_{2}$ are of type $F_{\alpha \delta}$ and measure zero and (ii) there exist disjoint sets $F_{1}$ and $F_{2}$ of type $F_{\sigma}$ such that $E_{1} \subset F_{1}$ and $E_{2} \subset F_{2}$.
\end{abstract}

\section{Introduction}

In [4] and [2], the following theorems are proved:

Theorem I. (Theorem 2 of [4]). Let $E_{1}$ and $E_{2}$ be disjoint subsets of $\mathbb{R}$. There exists a function $f: \mathbb{R} \rightarrow \mathbb{R}$ such that

$$
E_{1}=\left\{x: f_{-}^{\prime}(x)=+\infty\right\} \text { and } E_{2}=\left\{x: f_{-}^{\prime}(x)=-\infty\right\}
$$

if and only if $m\left(E_{1}\right)=m\left(E_{2}\right)=0$, where $f_{-}^{\prime}$ is the left-hand derivative and $m$ denotes the Lebesgue measure.

Analogously for the right-hand derivative.

Theorem II. (The main theorem of [2]). Let $E_{1}$ and $E_{2}$ be disjoint subsets of $\mathbb{R}$. There exists a function $f: \mathbb{R} \rightarrow \mathbb{R}$ such that

$$
E_{1}=\left\{x: f^{\prime}(x)=+\infty\right\} \text { and } E_{2}=\left\{x: f^{\prime}(x)=-\infty\right\}
$$

if and only if

Key Words: one-sided derivatives, $F_{\sigma}$-sets, $G_{\delta}$-sets

Mathematical Reviews subject classification: 26A24

Received by the editors October 7,1996 
(i) $E_{1}$ and $E_{2}$ are of type $F_{\sigma \delta}$ and of measure zero, and

(ii) there exist disjoint sets $F_{1}$ and $F_{2}$ of type $F_{\sigma}$ such that $E_{1} \subset F_{1}$ and $E_{2} \subset F_{2}$.

In the present paper we prove analogous theorems for the one-sided derivatives of continuous functions. To prove these results we use the construction from [2].

We shall apply the following notations:

$D^{-} f(x), D_{-} f(x), D^{+} f(x), D_{+} f(x)$ - the upper left-hand, lower left-hand, upper right-hand, lower right-hand Dini derivatives of a function $f$ at a point $x$;

$\int_{A} f(x) d x$ - the Lebesgue integral of $f$ on the set $A$;

$C_{f}$ - the set of all continuity points of the function $f$;

$D_{f}$ - the set of all discontinuity points of the function $f$;

\section{Main results}

Theorem 1 (Main Theorem).

1) Let $f: \mathbb{R} \rightarrow \mathbb{R}$ be a continuous function and let

$$
E_{1}=\left\{x: f_{+}^{\prime}(x)=+\infty\right\} \text { and } E_{2}=\left\{x: f_{+}^{\prime}(x)=-\infty\right\} .
$$

Then we have

(i) $E_{1}$ and $E_{2}$ are of type $F_{\sigma \delta}$ and of measure zero;

(ii) There exist disjoint $F_{\sigma}$-type sets $F_{1}$ and $F_{2}$ such that $E_{1} \subset F_{1}$ and $E_{2} \subset F_{2}$.

2) Let $E_{1}$ and $E_{2}$ be subsets of $\mathbb{R}$ that satisfy the following conditions:

(i) $E_{1}$ and $E_{2}$ are of type $F_{\sigma \delta}$ and of measure zero;

(ii) There exist disjoint $F_{\sigma}$-type sets $F_{1}$ and $F_{2}$ such that $E_{1} \subset F_{1}$ and $E_{2} \subset F_{2}$.

Then there exists a continuous function $f: \mathbb{R} \rightarrow \mathbb{R}$ such that

$$
E_{1}=\left\{x: f_{+}^{\prime}(x)=+\infty\right\} \text { and } E_{2}=\left\{x: f_{+}^{\prime}(x)=-\infty\right\} .
$$

Moreover, if $x \notin E_{1} \cup E_{2}$ then

$$
D_{+} f(x)<+\infty ; \quad D^{+} f(x)>-\infty ; \quad D_{-} f(x)<+\infty ; \quad D^{-} f(x)>-\infty .
$$


To prove this theorem we shall need the following five lemmas.

Lemma 1. Let $f: \mathbb{R} \rightarrow \mathbb{R}$. Then

$$
\left\{x: f_{+}^{\prime}(x)=+\infty\right\} \subset F_{1} \text { and }\left\{x: f_{+}^{\prime}(x)=-\infty\right\} \subset F_{2},
$$

where $F_{1} \cap F_{2}=\emptyset, F_{i}=A_{i} \backslash B_{i}, A_{i} \in F_{\sigma}$ and $B_{i} \subset D_{f}$ for $i=1,2$.

Proof. We have

$$
\left\{x: f_{+}^{\prime}(x)=+\infty\right\}=\left\{x: D_{+} f(x)=+\infty\right\}=\cap_{n=1}^{\infty}\left\{x: D_{+} f(x)>n\right\}
$$

and

$$
\left\{x: f_{+}^{\prime}(x)=-\infty\right\}=\left\{x: D^{+} f(x)=-\infty\right\}=\cap_{n=1}^{\infty}\left\{x: D^{+} f(x)<-n\right\} .
$$

Hence

$$
\left\{x: f_{+}^{\prime}(x)=+\infty\right\} \subset\left\{x: D_{+} f(x)>n\right\}, \quad n \in \mathbb{N}
$$

and

$$
\left\{x: f_{+}^{\prime}(x)=-\infty\right\} \subset\left\{x: D^{+} f(x)<-n\right\}, \quad n \in \mathbb{N} .
$$

Of course

$$
\left\{x: D_{+} f(x)>n\right\} \cap\left\{x: D^{+} f(x)<-n\right\}=\emptyset .
$$

By Lemma 8 of [5],

$$
\left\{x: D_{+} f(x) \leq n\right\} \cap C_{f} \in G_{\delta} \quad \text { and } \quad\left\{x: D^{+} f(x) \geq-n\right\} \cap C_{f} \in G_{\delta} .
$$

Hence

$$
\left\{x: D_{+} f(x)>n\right\} \cup D_{f} \in F_{\sigma} \quad \text { and } \quad\left\{x: D^{+} f(x)<-n\right\} \cup D_{f} \in F_{\sigma} .
$$

We have

$$
\left\{x: D_{+} f(x)>n\right\}=\left[\left\{x: D_{+} f(x)>n\right\} \cup D_{f}\right] \backslash\left[D_{f} \backslash\left\{x: D_{+} f(x)>n\right\}\right]
$$

and

$$
\left\{x: D^{+} f(x)<-n\right\}=\left[\left\{x: D^{+} f(x)<-n\right\} \cup D_{f}\right] \backslash\left[D_{f} \backslash\left\{x: D^{+} f(x)<-n\right\}\right] .
$$

Denoting

- $F_{1}=\left\{x: D_{+} f(x)>n\right\}$,

- $F_{2}=\left\{x: D^{+} f(x)<-n\right\}$, 
- $A_{1}=\left\{x: D_{+} f(x)>n\right\} \cup D_{f}$,

- $A_{2}=\left\{x: D^{+} f(x)<-n\right\} \cup D_{f}$,

we obtain our assertion.

Lemma 2. Let $u: \mathbb{R} \rightarrow \mathbb{R}$ be a nonnegative integrable function, let $M$ be a set of measure zero and $L^{*}=\cup_{j=1}^{\infty}\left(a_{j}, b_{j}\right)$ such that $M \subset L^{*}$. For every interval $\left(a_{j}, b_{j}\right)$ we denote by

$$
\begin{aligned}
& \Delta_{j, n}=\left(a_{j}+\frac{b_{j}-a_{j}}{2^{n+1}}, a_{j}+\frac{b_{j}-a_{j}}{2^{n}}\right) \\
& \Delta_{j, n}^{\prime}=\left(b_{j}-\frac{b_{j}-a_{j}}{2^{n}}, b_{j}-\frac{b_{j}-a_{j}}{2^{n+1}}\right)
\end{aligned}
$$

Then for each positive integer $k$, there exists an open set $L_{k} \subset L^{*}$ such that

1) $M \subset L_{k}$;

2) $\int_{L_{k} \cap \Delta_{j, n}} u(x) d x<\frac{1}{2^{k}} \cdot \frac{m\left(\Delta_{j, n}\right)}{2}$ and $\int_{L_{k} \cap \Delta_{j, n}^{\prime}} u(x) d x<\frac{1}{2^{k}} \cdot \frac{m\left(\Delta_{j, n}^{\prime}\right)}{2}$.

3) $\int_{L_{k} \cap\left(a_{j}, b_{j}\right)} u(x) d x<\frac{1}{2^{k}}\left(b_{j}-a_{j}\right)$;

4) If $x_{o} \notin L^{*}$ then for every $h$, we have

$$
\int_{L_{k}^{h}} u(x) d x<\frac{1}{2^{k}}|h|, \quad \text { where }
$$

$L_{k}^{h}=\left[x_{o}, x_{o}+h\right] \cap L_{k}$ for $h>0$ and $L_{k}^{h}=\left[x_{o}+h, x_{o}\right] \cap L_{k}$ for $h<0$.

5) For every $x_{o} \in L^{*}$ there exists $h>0$ (respectively $h<0$ ) such that

$$
\begin{gathered}
\int_{L_{k}^{h}} u(x) d x<\frac{1}{2^{k-1}}|h| \text { and } \\
\left(x_{o}, x_{o}+h\right) \subset L^{*}\left(\text { respectively }\left(x_{o}+h, x_{o}\right) \subset L^{*}\right) .
\end{gathered}
$$

Proof. 1)-4) See Codyks' paper [2] (pp. 435-436).

5) Since $x_{o} \in L^{*}$, there exists $j$ such that $x_{o} \in\left(a_{j}, b_{j}\right)$. Let $\Delta_{j, n}$ and $\Delta_{j, n}^{\prime}$ be the intervals defined in our hypothesis. Suppose that

$$
\Delta_{j, n}=\left(p_{n}^{j}, q_{n}^{j}\right) \quad \text { and } \quad \Delta_{j, n}^{\prime}=\left(r_{n}^{j}, t_{n}^{j}\right) .
$$


Of course $p_{n}^{j}=q_{n+1}^{j}$ and $t_{n}^{j}=r_{n+1}^{j}$, and also

$$
m\left(\Delta_{j, n+1}\right)=\frac{1}{2} m\left(\Delta_{j, n}\right), \quad m\left(\Delta_{j, n+1}^{\prime}\right)=\frac{1}{2} m\left(\Delta_{j, n}^{\prime}\right) .
$$

If $x_{o} \in\left(a_{j}, \frac{a_{j}+b_{j}}{2}\right]$ then there exists $h \geq \frac{1}{2}\left(b_{j}-a_{j}\right)$ such that $\left[x_{o}, x_{o}+h\right] \subset$ $\left(a_{j}, b_{j}\right)$. From condition 3$)$ we obtain

$$
\int_{L_{k}^{h}} u(x) d x \leq \int_{L_{k} \cap\left(a_{j}, b_{j}\right)} u(x) d x<\frac{1}{2^{k}}\left(b_{j}-a_{j}\right),
$$

hence

$$
\int_{L_{k}^{h}} u(x) d x<\frac{1}{2^{k-1}} h
$$

If $x_{o} \in\left(\frac{a_{j}+b_{j}}{2}, b_{j}\right)$ then there exists $\Delta_{j, n}^{\prime}$ such that $x_{o} \in{\overline{\Delta_{j, n}^{\prime}}}^{\prime}$. Suppose that

$$
\left(t_{n}^{j}-x_{o}\right) \geq \frac{1}{2} m\left(\Delta_{j, n}^{\prime}\right) .
$$

Then there exists $h \geq \frac{1}{2} m\left(\Delta_{j, n}^{\prime}\right)$ such that $x_{o}+h \in \bar{\Delta}_{j, n}^{\prime}$. From condition 2) we obtain

$$
\int_{L_{k}^{h}} u(x) d x \leq \int_{L_{k} \cap \Delta_{j, n}^{\prime}} u(x) d x<\frac{1}{2^{k}} \cdot \frac{m\left(\Delta_{j, n}^{\prime}\right)}{2} .
$$

It follows that

$$
\int_{L_{k}^{h}} u(x) d x<\frac{1}{2^{k}} h \quad \text { so } \quad \int_{L_{k}^{h}} u(x) d x<\frac{1}{2^{k-1}} h .
$$

Suppose that

$$
\left(t_{n}^{j}-x_{o}\right)<\frac{1}{2} m\left(\Delta_{j, n}^{\prime}\right) .
$$

Then there exists $h>0$ such that $x_{o}+h \in \bar{\Delta}_{j, n+1}^{\prime}$ and $h \geq \frac{1}{2} m\left(\Delta_{j, n}^{\prime}\right)$.

By (1) we have

$$
h>\frac{1}{2} m\left(\Delta_{j, n+1}^{\prime}\right) .
$$

It follows that

$$
\int_{L_{k}^{h}} u(x) d x=\int_{\left[x_{o}, t_{n}^{j}\right] \cap L_{k}} u(x) d x+\int_{\left[t_{n}^{j}, x_{o}+h\right] \cap L_{k}} u(x) d x .
$$


From condition 2) we obtain

$$
\int_{\left[x_{o}, t_{n}^{j}\right] \cap L_{k}} u(x) d x \leq \int_{L_{k} \cap \Delta_{j, n}^{\prime}} u(x) d x<\frac{1}{2^{k}} \cdot \frac{m\left(\Delta_{j, n}^{\prime}\right)}{2} .
$$

and

$$
\int_{\left[t_{n}^{j}, x_{o}+h\right]} u(x) d x \leq \int_{L_{k} \cap \Delta_{j, n+1}^{\prime}} u(x) d x<\frac{1}{2^{k}} \cdot \frac{m\left(\Delta_{j, n+1}^{\prime}\right)}{2} .
$$

Hence

$$
\int_{L_{k}^{h}} u(x) d x<\frac{1}{2^{k}}\left(\frac{m\left(\Delta_{j, n}^{\prime}\right)}{2}+\frac{m\left(\Delta_{j, n+1}^{\prime}\right)}{2}\right),
$$

in consequence

$$
\int_{L_{k}^{h}} u(x) d x<\frac{2}{2^{k}} h=\frac{1}{2^{k-1}} h .
$$

Analogously it follows that there exists $h<0$ that satisfies condition 5).

Lemma 3. Let $E^{1}, E^{2}, H_{1}, H_{2}$ be sets such that $E^{i} \in F_{\sigma \delta}, H_{i} \in F_{\sigma}, m\left(E^{i}\right)=$ $0, E^{i} \subset H_{i}(i=1,2)$ and $H_{1} \cap H_{2}=\emptyset$. Let

$$
E^{i}=\cap_{n=1}^{\infty} E_{n}^{i}, \quad E_{n}^{i}=\cup_{k=1}^{\infty} E_{n, k}^{i}, \quad H_{i}=\cup_{k=1}^{\infty} F_{k}^{i},
$$

where $E_{n, k}^{i}$ and $F_{k}^{i}$ are closed sets, $i=1,2$. Let $G_{n}$ be an open set such that $E^{1} \cup E^{2} \subset G_{n}$ and $m\left(G_{n}\right)<\frac{1}{2^{n}}$, where $n=1,2, \ldots$ We may additionally suppose that

$G_{n+1} \subset G_{n}, \quad E_{n}^{i} \subset G_{n}, \quad F_{k}^{i} \subset F_{k+1}^{i}, \quad E_{n, k}^{i} \subset E_{n, k+1}^{i}, \quad E_{n+1, k}^{i} \subset E_{n_{k}}^{i} \subset F_{k}^{i}$.

Let us denote by $C_{k}^{1}$ and $C_{k}^{2}$ some disjoint open sets such that $F_{k}^{i} \subset C_{k}^{i}$ (i= $1,2)$. Let $u: \mathbb{R} \rightarrow \mathbb{R}$ be defined as follows:

$$
u(x)=\sum_{n=1}^{\infty} u_{n}(x), \text { where } u_{n}(x)= \begin{cases}1 & \text { if } x \in G_{n} \\ 0 & \text { if } x \notin G_{n}\end{cases}
$$

(clearly $u$ is an integrable function). Then there exist some sets $e_{n}^{1} \subset E_{n}^{1}$ and $e_{n}^{2} \subset E_{n}^{2}$ where

$$
e_{n}^{i}=\cup_{k=1}^{\infty} e_{n, k}^{i}, \quad e_{n, k}^{i} \in F_{\sigma} \cap G_{\delta} \quad(i=1,2),
$$

such that the sets $e_{n, k}^{i}$ satisfy the following conditions: 
1) $e_{n, 1}^{i}=E_{n, 1}^{i} \subset C_{1}^{i}, e_{n, k}^{i} \subset e_{n, k+1}^{i}, E^{i} \cap E_{n, k}^{i} \subset e_{n, k}^{i} \subset E_{n, k}^{i} \subset C_{k}^{i}$.

2) For every positive integer $k \geq 2$ there exist some open sets $g_{n, k}^{*}, g_{n, k}$, and for $k=1$ there exists the open set $g_{n, 1}$, such that:

a) $G_{n}=g_{n, 1} \supset e_{n, 1}^{i}$;

b) $g_{n, k-1} \backslash\left(e_{n, k-1}^{1} \cup e_{n, k-1}^{2}\right) \supset g_{n, k}^{*} \supset g_{n, k} \supset\left(e_{n, k}^{i} \backslash e_{n, k-1}^{i}\right)$;

c) the points of the set $e_{n, 1}^{i}$ are the points of density of the set $g_{n, 1} \backslash g_{n, 2}^{*}$, while for $k \geq 2$, the points of the set $e_{n, k}^{i} \backslash e_{n, k-1}^{i}$ are the points of density of the set $g_{n, k} \backslash g_{n, k+1}^{*}$;

d) for every component I of the set $g_{n, k}^{*}$ we have

$$
\int_{I \cap g_{n, k}} u(x) d x<\frac{1}{2^{k}} m(I)
$$

e) for $x_{o} \in g_{n, k}^{*}$ there exists $h>0$ such that $\left[x_{o}, x_{o}+h\right] \subset g_{n, k}^{*}$ and there exists $h<0$ such that $\left[x_{o}+h, x_{o}\right] \subset g_{n, k}^{*}$, and

$$
\int_{g_{n, k}^{h}} u(x) d x<\frac{1}{2^{k-1}}|h|,
$$

where $g_{n, k}^{h}=g_{n, k} \cap\left[x_{o}, x_{o}+h\right]$ for $h>0$ and $g_{n, k}^{h}=g_{n, k} \cap\left[x_{o}+h, x_{o}\right]$ for $h<0$;

f) if $c_{o} \notin g_{n, k}^{*}$ then for every $h$,

$$
\int_{g_{n, k}^{h}} u(x) d x<\frac{1}{2^{k}}|h| .
$$

Proof. For a), b), c), d), f) see [2] (pp. 436-440). Condition e) follows from Lemma 2, 5).

Corollary 1. ([2], p.440).

1) The sets $e_{n}^{1}$ and $e_{n}^{2}$ are of type $F_{\sigma}$;

2) $E^{i} \subset e_{n}^{i} \subset E_{n}^{i} \quad(i=1,2)$;

3) The points of the set $e_{n}^{1}$ are the density points of the set

$$
\Omega_{n}^{1}=\cup_{k=1}^{\infty}\left[\left(g_{n, k} \backslash g_{n, k+1}^{*}\right) \cap C_{k}^{1}\right] ;
$$

The points of the set $e_{n}^{2}$ are the density points of the set

$$
\Omega_{n}^{2}=\cup_{k=1}^{\infty}\left[\left(g_{n, k} \backslash g_{n, k+1}^{*}\right) \cap C_{k}^{2}\right] ;
$$


4) $\Omega_{n}^{1} \cap \Omega_{n}^{2}=\emptyset$.

Lemma 4. (Lemma 6 of [2], p. 440). Let $K \in G_{\delta}$ and $K \subset G$, where $G$ is an open set. Then $K=\cap_{j=1}^{\infty} K^{(j)}$, where $K^{(j)}$ is an open set such that

$$
K^{(j+1)} \subset K^{(j)} \subset G, \quad \text { and } \bar{K}^{(j+1)} \subset K^{(j)} \cup \bar{K} .
$$

Corollary 2. The sets $e_{n, 1}^{i}$ and $e_{n, k}^{i} \backslash e_{n, k-1}^{i}(n=1,2, \ldots, k=2,3, \ldots)$ from Lemma 3 are of the form:

1) $e_{n, 1}^{i}=\cap_{j=1}^{\infty} G_{n, 1}^{(j)_{i}}$, where $G_{n, 1}^{(j)_{i}}$ are open sets satisfying

$$
\bar{G}_{n, 1}^{(j+1)_{i}} \subset G_{n, 1}^{(j)_{i}} \subset g_{n, 1} ;
$$

2) $e_{n, k}^{i} \backslash e_{n, k-1}^{i}=\cap_{j=1}^{\infty} G_{n, k}^{(j)_{i}}$, where $G_{n, k}^{(j)_{i}}$ are open sets satisfying

$$
G_{n, k}^{(j+1)_{i}} \subset G_{n, k}^{(j)_{i}} \subset g_{n, k} \quad \text { and } \quad \bar{G}_{n, k}^{(j+1)_{i}} \subset G_{n, k}^{(j)_{i}} \cup E_{n, k}^{i} .
$$

Proof. See Lemma 4.

Lemma 5. Let the functions $v_{n}^{(1)}(x)$ and $v_{n}^{(2)}(x)$ be defined by

$$
v_{n}^{(1)}(x)= \begin{cases}1 & \text { if } x \in G^{(j)_{1}} \backslash G^{(j+1)_{1}} \\ +\infty & \text { if } x \in \cap_{j=1}^{\infty} G_{n}^{(j)_{1}} \\ 0 & \text { elsewhere }\end{cases}
$$

and

$$
v_{n}^{(2)}(x)= \begin{cases}-1 & \text { if } x \in G^{(j)_{2}} \backslash G^{(j+1)_{2}} \\ -\infty & \text { if } x \in \cap_{j=1}^{\infty} G_{n}^{(j)_{2}} \\ 0 & \text { elsewhere }\end{cases}
$$

where $G_{n}^{(j)_{i}}=\cup_{k=1}^{\infty} G_{n, k}^{(j)_{i}}$ and $G_{n, k}^{(j)_{i}}$ are the sets from Corollary 2. We have

$$
e_{n}^{i} \subset G_{n}^{(j)_{i}} \subset G_{n} .
$$

Let

$$
w_{n}^{(1)}(x)= \begin{cases}\min \left[v_{n}^{(1)}(x), u(x)\right] & \text { if } x \in \Omega_{n}^{1} \\ 0 & \text { elsewhere }\end{cases}
$$


and

$$
w_{n}^{(2)}(x)= \begin{cases}\max \left[v_{n}^{(2)}(x),-u(x)\right] & \text { if } x \in \Omega_{n}^{2} \\ 0 & \text { elsewhere }\end{cases}
$$

where $u, \Omega_{n}^{1}$ and $\Omega_{n}^{2}$ are defined in Lemma 3. Then the functions

$$
W_{n}^{(1)}(x)=\int_{0}^{x} w_{n}^{(1)}(t) d t \quad \text { and } \quad W_{n}^{(2)}(x)=\int_{0}^{x} w_{n}^{(2)}(t) d t
$$

have the following properties:

1) $0 \leq D_{+} W_{n}^{(1)}(x)<+\infty$ and $0 \leq D_{-} W_{n}^{(1)}(x)<+\infty$ for $x \notin E_{n}^{1}$,

2) $0 \leq D^{+} W_{n}^{(1)}(x)<+\infty$ and $0 \leq D^{-} W_{n}^{(1)}(x)<+\infty$ for $x \in E^{2}$,

3) $-\infty<D^{+} W_{n}^{(2)}(x) \leq 0$ and $-\infty<D^{-} W_{n}^{(2)}(x) \leq 0$ for $x \notin E_{n}^{2}$,

4) $-\infty<D_{+} W_{n}^{(2)}(x) \leq 0$ and $-\infty<D_{-} W_{n}^{(2)}(x) \leq 0$ for $x \in E_{n}^{1}$.

The sets $E^{i}$ and $E_{n}^{i}(i=1,2)$ are defined in Lemma 3 and Corollary 1.

Proof. Consider first the case where $x \in g_{n, k}^{*}$ for every $k \geq 2$. Let $k_{1} \geq 2$. Then there exists a component $I_{1}$ of the set $g_{n, k_{1}}^{*}$ such that $x \in I_{1}$. By the definition of $W_{n}^{(1)}$, for any $h>0$ with $[x, x+h] \subset I_{1}$, and for any $h<0$ with $[x+h, x] \subset I_{1}$ we have

$$
\int_{x}^{x+h} w_{n}^{(1)}(t) d t=\int_{g_{n, k_{1}}^{h}} w_{n}^{(1)}(t) d t \leq \int_{g_{n, k_{1}}^{h}} u(t) d t
$$

where

$$
g_{n, k_{1}}^{h}=[x, x+h] \cap g_{n, k_{1}} \quad \text { for } \quad h>0,
$$

and

$$
g_{n, k_{1}}^{h}=[x+h, x] \cap g_{n, k_{1}} \quad \text { for } \quad h<0 .
$$

By Lemma 3 , e), there exist $h_{1}>0$ with $\left[x, x+h_{1}\right] \subset I_{1}$, and $h_{1}^{\prime}<0$ with $\left[x+h_{1}^{\prime}, x\right] \subset I_{1}$ such that

$$
\int_{x}^{x+h_{1}} w_{n}^{(1)}(t) d t \leq \int_{g_{n, k_{1}}^{h_{1}}} u(t) d t<\frac{1}{2^{k_{1}-1}}\left|h_{1}\right|,
$$

and

$$
\int_{x}^{x+h_{1}^{\prime}} w_{n}^{(1)}(t) d t \leq \int_{g_{n, k_{1}}^{h_{1}^{\prime}}} u(t) d t<\frac{1}{2^{k_{1}-1}}\left|h_{1}^{\prime}\right|
$$


hence

$$
-\frac{1}{2^{k_{1}-1}}\left|h_{1}^{\prime}\right|<\int_{x}^{x+h_{1}^{\prime}} w_{n}^{(1)}(t) d t .
$$

By this inequality we have

$$
0 \leq \frac{W_{n}^{(1)}\left(x+h_{1}\right)-W_{n}^{(1)}(x)}{h_{1}}=\frac{\int_{x}^{x+h_{1}} w_{n}^{(1)}(t) d t}{h_{1}}<\frac{1}{2^{k_{1}-1}},
$$

and

$$
0 \leq \frac{W_{n}^{(1)}\left(x+h_{1}^{\prime}\right)-W_{n}^{(1)}(x)}{h_{1}^{\prime}}=\frac{\int_{x}^{x+h_{1}^{\prime}} w_{n}^{(1)}(t) d t}{h_{1}^{\prime}}<\frac{1}{2^{k_{1}-1}} .
$$

Let $k_{2}>k_{1}$. Then there exists a component $I_{2}$ of the set $g_{n, k_{2}}^{*}$ such that $x \in I_{2}$. Similarly as above, we obtain that there exist $h_{2}>0$ and $h_{2}^{\prime}<0$ such that

$$
0 \leq \frac{W_{n}^{(1)}\left(x+h_{2}\right)-W_{n}^{(1)}(x)}{h_{2}}<\frac{1}{2^{k_{2}-1}},
$$

and

$$
0 \leq \frac{W_{n}^{(1)}\left(x+h_{2}^{\prime}\right)-W_{n}^{(1)}(x)}{h_{2}^{\prime}}<\frac{1}{2^{k_{2}-1}}
$$

and so on.

By Lemma 3 it follows that $m\left(I_{n}\right) \rightarrow 0$ if $k_{n} \rightarrow \infty$. Then $h_{n} \rightarrow 0$ and $h_{n}^{\prime} \rightarrow$ 0 . Finally if $x \in g_{n, k}^{*}$ for every $k$ then $D_{+} W_{n}^{(1)}(x)=0$ and $D_{-} W_{n}^{(1)}(x)=0$.

Consider the case where $x \notin E_{n}^{1}$ and $x \notin g_{n, k}^{*}$ for $k \geq k_{o}$, where $k_{o}$ is a positive integer. From Lemma $3, E_{n}^{1}=\cup_{k=1}^{\infty} E_{n, k}^{1}$ and $E_{n}^{1}=e_{n, 1}^{1}$. Since $x \notin E_{n}^{1}$ it follows that $x \notin E_{n, k}^{1}$ for every $k$. Hence $x \notin e_{n, 1}^{1}$. By Corollary 2, $e_{n, 1}^{1}=\cap_{j=1}^{\infty} G_{n, 1}^{(j)_{1}}$. It follows that there exists some $j_{1}$ such that $x \notin G_{n, 1}^{\left(j_{1}\right)_{1}}$. Since $x \notin E_{n}^{1} \supset e_{n}^{1} \supset e_{n, 2}^{1} \backslash e_{n, 1}^{1}$ (see Lemma 3), we obtain that $x \notin e_{n, 2}^{1} \backslash e_{n, 1}^{1}$. By Corollary 2, $e_{n, 2}^{1} \backslash e_{n, 1}^{1}=\cap_{j=1}^{\infty} G_{n, 2}^{(j)_{1}}$. Then there exists some $j_{2}$ such that $x \notin G_{n, 2}^{\left(j_{2}\right)_{1}}$.

Continuing the above procedure we have

$$
x \notin e_{n, k_{o}-1}^{1} \backslash e_{n, k_{o}-2}^{1} \subset E_{n}^{1},
$$

and there exists $j_{k_{o}-1}$ such that $x \notin G_{n, k_{o}-1}^{\left(j_{k_{o}-1}\right)_{1}}$. Let

$$
j_{o}=\max \left(j_{1}, j_{2}, \ldots, j_{k_{o}-1}\right\} .
$$


From the properties of the sets $G_{n, k}^{(j)_{i}}$ (see Corollary 2), we get

$$
x \notin \cup_{k<k_{o}} G_{n, k}^{\left(j_{o}\right)_{1}} \cup E_{n}^{1} .
$$

This implies the fact that: the distance of the point $x$ to the set $\cup_{k<k_{o}} \bar{G}_{n, k}^{\left(j_{o}+1\right)_{1}}$ is positive, therefore to $\cup_{k<k_{o}} G_{n, k}^{\left(j_{o}+1\right)_{1}}$ the distance is positive too. Then there exists an open interval $\Delta$ such that $x \in \Delta$ and

$$
\Delta \cap\left(\cup_{k<k_{o}} G_{n, k}^{\left(j_{o}+1\right)_{1}}\right)=\emptyset .
$$

There exist $h>0$ with $[x, x+h] \subset \Delta$, and $h<0$ with $[x+h, x] \subset \Delta]$ such that

$$
\begin{gathered}
0 \leq \frac{W_{n}^{(1)}(x+h)-W_{n}^{(1)}(x)}{h}=\frac{\int_{x}^{x+h} w_{n}^{(1)}(t) d t}{h}= \\
=\frac{\int_{g_{n, k_{o}}^{h}} w_{n}^{(1)}(t) d t}{|h|}+\frac{\int_{\sim g_{n, k_{o}}^{h}} w_{n}^{(1)}(t) d t}{|h|},
\end{gathered}
$$

where $\sim g_{n, k_{o}}^{h}=[x, x+h] \backslash g_{n, k_{o}}^{h}$. Since $x \notin g_{n, k}^{*}$, by Lemma $\left.3, \mathrm{f}\right)$, we have

$$
\frac{\int_{g_{n, k_{o}}^{h}} w_{n}^{(1)}(t) d t}{|h|} \leq \frac{\int_{g_{n, k_{o}}^{h}} u(t) d t}{|h|}<\frac{1}{2^{k_{o}}}<1 .
$$

From Lemma 3 we obtain the following fact: if $t \notin g_{n, k_{o}}$ then $t \notin g_{n, k}$ for $k \geq k_{o}$, therefore $t \notin G_{n, k}^{(j)_{1}}$ for $k \geq k_{o}$ and $j \geq 1$ (see Corollary 2). If $t \in[x, x+h]$ then $t \notin \cup_{k<k_{o}} G_{n, k}^{\left(j_{o}+1\right)_{1}}$. It follows that if $t \in \sim g_{n, k_{o}}^{h}$ then

$$
t \notin G_{n}^{\left(j_{o}+1\right)_{1}}=\cup_{k=1}^{\infty} G_{n, k}^{\left(j_{o}+1\right)_{1}} \quad \text { and } \quad 0 \leq w_{n}^{(1)}(t) \leq j_{o} .
$$

This implies that

$$
\frac{\int_{g_{n, k_{o}}^{h}} w_{n}^{(1)}(t) d t}{|h|} \leq j_{o}
$$

We have

$$
\begin{aligned}
& 0 \leq D^{+} W_{n}^{(1)}(x)<j_{o}+1, \\
& 0 \leq D_{+} W_{n}^{(1)}(x)<j_{o}+1, \\
& 0 \leq D^{-} W_{n}^{(1)}(x)<j_{o}+1, \\
& 0 \leq D_{-} W_{n}^{(1)}(x)<j_{o}+1 .
\end{aligned}
$$


Hence, for $x \notin E_{n}^{1}$ we have

$$
0 \leq D_{+} W_{n}^{(1)}(x)<+\infty \quad \text { and } \quad 0 \leq D_{-} W_{n}^{(1)}(x)<+\infty .
$$

Consider the case where $x \in E^{2}$. By Lemma 3, there exists some $k$ such that $x \in E_{n, k}^{2}$. By Lemma 3,1), we have $E^{2} \cap E_{n, k}^{2} \subset e_{n, k}^{2}$, hence $x \in e_{n}^{2}$. Then there exists some $k_{o}$ such that $x \in e_{n, k_{o}-1}^{2}$. Since

$$
g_{n, k_{o}}^{*} \subset g_{n, k_{o}-1} \backslash\left(e_{n, k_{o}-1}^{1} \cup e_{n, k_{o}-1}^{2}\right),
$$

it follows that $x \notin g_{n, k_{o}}^{*}$ and $x \notin E_{n}^{1}$. From this fact we have

$$
0 \leq D^{+} W_{n}^{(1)}(x)<+\infty \quad \text { and } \quad 0 \leq D^{-} W_{n}^{(1)}(x)<+\infty .
$$

Analogously we can prove 3) and 4).

Proof. [Proof of Theorem 1]

1) This follows from Theorem 1 of [3] and Lemma 1 , where $B_{1}=B_{2}=\emptyset$.

2) The proof is as that of Theorem 2 of [2] (see pp. 445-449), using properties 1)-4) from Lemma 5.

Theorem 2. Let $f: \mathbb{R} \rightarrow \mathbb{R}$ be a function such that every point $x \in D_{f}$ is isolated in $D_{f}$. Then there exist $F_{1}, F_{2} \in F_{\sigma}$ with $F_{1} \cap F_{2}=\emptyset$ such that

$$
\left\{x: f_{+}^{\prime}(x)=+\infty\right\} \subset F_{1} \quad \text { and } \quad\left\{x: f_{+}^{\prime}(x)=-\infty\right\} \subset F_{2} .
$$

Proof. This theorem follows from Lemma 1. Since $B_{1} \subset D_{f}$ and $B_{2} \subset D_{f}$ it follows that every point of $B_{1}$ or $B_{2}$ is isolated in $B_{1}, B_{2}$. The sets $B_{1}$ and $B_{2}$ are at most countable. From the definition of an isolated point we have that:

$$
\mathbb{R} \backslash B_{1}=\cup_{n=1}^{\infty} I_{n}^{1} \cup P_{1} \text { and } \quad \mathbb{R} \backslash B_{2}=\cup_{n=1}^{\infty} I_{n}^{2} \cup P_{2},
$$

where $I_{n}^{1}, I_{n}^{2}$ are intervals such that $I_{k}^{i} \cap I_{1}^{i}=\emptyset$ for $k \neq 1(i=1,2)$ and $I_{k}^{i}=\left(a_{k}^{i}, b_{k}^{i}\right)$ with $a_{k}^{i}, b_{k}^{i} \in B_{i}, P_{i}$ is the set of all accumulation points of $B_{i}$. Of course $\cup_{n=1}^{\infty} I_{n}^{i}$ is an open set and $P_{i}$ are closed sets. Hence $\mathbb{R} \backslash B_{1} \in F_{\sigma}$ and $\mathbb{R} \backslash B_{2} \in F_{\sigma}$. It follows that $F_{i} \in A_{i} \backslash B_{i} \in F_{\sigma}(i=1,2)$. This proves our theorem.

Remark 1. From Theorem 1 of [3] and our Theorem 2, it follows that in Theorem 1 we may replace condition " $f: \mathbb{R} \rightarrow \mathbb{R}, f$ a continuous function" with " $f: \mathbb{R} \rightarrow \mathbb{R}$ such that every point $x \in D_{f}$ is an isolated point of $D_{f}$ ". 


\section{Infinite one-sided derivatives and Baire functions}

Theorem 3. Let $f: \mathbb{R} \rightarrow \mathbb{R}$ be a Baire function of $\alpha$ class $\left(f \in \mathcal{B}_{\alpha}\right)$. Then there exist some sets $F_{i}, i=1,2,3,4$, such that

$$
\begin{aligned}
& \left\{x: f_{+}^{\prime}(x)=+\infty\right\} \subset F_{1}, \quad\left\{x: f_{+}^{\prime}(x)=-\infty\right\} \subset F_{2}, \\
& \left\{x: f_{-}^{\prime}(x)=+\infty\right\} \subset F_{3}, \quad\left\{x: f_{-}^{\prime}(x)=-\infty\right\} \subset F_{4},
\end{aligned}
$$

where $F_{2}, F_{4} \in F_{\alpha+1}, F_{1}, F_{3} \in G_{\alpha+1}$, and $F_{1} \cap F_{2}=\emptyset, F_{3} \cap F_{4}=\emptyset$.

Proof. The case $\alpha=0$ follows from Theorem 1, 1).

Let $\alpha>0$. We have

$$
\left\{x: f_{+}^{\prime}(x)=+\infty\right\}=\left\{x: D_{+} f(x)=+\infty\right\}=\cap_{p=1}^{\infty}\left\{x: D_{+} f(x)>p\right\}
$$

and

$$
\left\{x: f_{+}^{\prime}(x)=-\infty\right\}=\left\{x: D^{+} f(x)=-\infty\right\}=\cap_{p=1}^{\infty}\left\{x: D^{+} f(x)<-p\right\} .
$$

Hence

$$
\left\{x: f_{+}^{\prime}(x)=+\infty\right\} \subset\left\{x: D^{+} f(x)>p\right\}
$$

and

$$
\left\{x: f_{+}^{\prime}(x)=-\infty\right\} \subset\left\{x: D^{+} f(x)<-p\right\},
$$

where $p \in \mathbb{N}$. Let

$$
\begin{aligned}
F_{m, n}(x) & =\sup _{\frac{1}{m} \leq h \leq \frac{1}{n}} \frac{f(x+h)-f(x)}{h}, \\
H_{m, n}(x) & =\inf _{\frac{1}{m} \leq h \leq \frac{1}{n}} \frac{f(x+h)-f(x)}{h}, \\
F_{n}(x) & =\sup _{0<h \leq \frac{1}{n}} \frac{f(x+h)-f(x)}{h}, \\
H_{n}(x) & =\inf _{0<h \leq \frac{1}{n}} \frac{f(x+h)-f(x)}{h} .
\end{aligned}
$$

We have

$$
\begin{gathered}
F_{n}(x)=\lim _{m \rightarrow \infty} F_{m, n}(x), \quad H_{n}(x)=\lim _{m \rightarrow \infty} H_{m, n}, \\
F_{n}(x) \geq F_{n+1}(x), \quad H_{n}(x) \leq H_{n+1}(x) .
\end{gathered}
$$


By [1], $F_{m, n} \in \mathcal{B}_{\alpha}$ and $H_{m, n} \in \mathcal{B}_{\alpha}$. Consequently $F_{n}, H_{n} \in \mathcal{B}_{\alpha+1}$. We prove that

$$
D^{+} f(x)=\lim _{n \rightarrow \infty} F_{n}(x) \quad \text { and } \quad D_{+} f(x)=\lim _{n \rightarrow \infty} H_{n}(x) .
$$

Let $y=\lim _{n \rightarrow \infty} F_{n}(x)$. Then there exist $y_{n}<F_{n}(x)$ such that $y_{n} \rightarrow y$ if $n \rightarrow \infty$. Since $y_{n}<F_{n}(x)$ and by the definition of $F_{n}(x)$, we know that there exists $h_{n} \in\left(0, \frac{1}{n}\right]$ such that

$$
F_{n}(x) \geq \frac{f\left(x+h_{n}\right)-f(x)}{h_{n}}>y_{n}
$$

Hence

$$
\lim _{n \rightarrow \infty} \frac{f\left(x+h_{n}\right)-f(x)}{h_{n}}=y \quad \text { and } \quad D^{+} f(x) \geq y .
$$

Let $z_{n}<D^{+} f(x), z_{n} \rightarrow D^{+} f(x)$. For every $n$ there exists $h_{n} \in\left(0, \frac{1}{n}\right]$ such that

$$
\frac{f\left(x+h_{n}\right)-f(x)}{h_{n}}>z_{n} .
$$

Hence $F_{n}(x)>z_{n}$ and $y=\lim _{n \rightarrow \infty} F_{n}(x) \geq D^{+} f(x)$. Finally $D^{+} f(x)=$ $\lim _{n \rightarrow \infty} F_{n}(x)$. Analogously $D_{+} f(x)=\lim _{n \rightarrow \infty} H_{n}(x)$.

From the definitions of the limits we have:

$$
D^{+} f(x)=\inf _{n}\left\{F_{n}(x)\right\} \quad \text { and } \quad D_{+} f(x)=\sup _{n}\left\{H_{n}(x)\right\} .
$$

Hence

$$
\begin{gathered}
\left\{x: D^{+} f(x)<-p\right\} \subset \cup_{n=1}^{\infty}\left\{x: F_{n}(x)<-p\right\}, \\
\left\{x: D_{+} f(x)>-p\right\} \subset \cup_{n=1}^{\infty}\left\{x: H_{n}(x)>p\right\} .
\end{gathered}
$$

Because $F_{n}, H_{n} \in \mathcal{B}_{\alpha+1}$, it follows that $\left\{x: F_{n}(x)<-p\right\} \in F_{\alpha+1}$ if $\alpha$ is even, and $\left\{x: H_{n}(x)>p\right\} \in G_{\alpha+1}$ if $\alpha$ is odd. It follows that the sets

$$
F_{1}=\cup_{n=1}^{\infty}\left\{x: F_{n}(x)<-p\right\} \quad \text { and } \quad F_{2}=\cup_{n=1}^{\infty}\left\{x: H_{n}(x)>p\right\}
$$

are identical. From the properties of $F_{n}$ and $H_{n}$ we have $F_{1} \cap F_{2}=\emptyset$.

For $F_{3}, F_{4}$ and the left-hand derivatives the proof is similar.

\section{References}

[1] S. Banach, Sur les fonctions derivees des fonctions mesurables, Fundam. Math. 3 (1922), 128-132. 
[2] M. Codyks, On the sets of points at which the derivative is suitable equal $+\infty$ and $\infty$, Mat. Sb. 43 (1957), no. 85, 429-450, in Russian.

[3] B. Kaczmarski, On the set of points at which a function has no one-sided derivative, Demonstratio Math. 18 (1985), no. 4, 1127-1141.

[4] B. Kaczmarski, On the set of points at which a function has infinite onesided derivative, Real Analysis Exchange 16 (1990-1991), no. 2, 421-424.

[5] Z. Zahorski, Sur la premiere derivée, Trans. Amer. Math. Soc. 69 (1950), $1-54$. 